2012s-17

\title{
Local Utility and Multivariate Risk Aversion
}

Arthur Charpentier, Alfred Galichon, Marc Henry

\begin{tabular}{c}
\hline Série Scientifique \\
Scientific Series
\end{tabular}

\author{
Montréal \\ Juin 2012
}

(C) 2012 Arthur Charpentier, Alfred Galichon, Marc Henry. Tous droits réservés. All rights reserved. Reproduction partielle permise avec citation du document source, incluant la notice (C)

Short sections may be quoted without explicit permission, if full credit, including (C) notice, is given to the source.
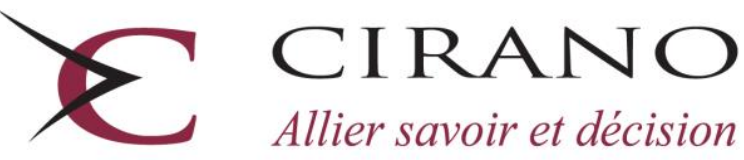

Allier savoir et décision

Centre interuniversitaire de recherche en analyse des organisations 


\section{CIRANO}

Le CIRANO est un organisme sans but lucratif constitué en vertu de la Loi des compagnies du Québec. Le financement de son infrastructure et de ses activités de recherche provient des cotisations de ses organisations-membres, d'une subvention d'infrastructure du Ministère du Développement économique et régional et de la Recherche, de même que des subventions et mandats obtenus par ses équipes de recherche.

CIRANO is a private non-profit organization incorporated under the Québec Companies Act. Its infrastructure and research activities are funded through fees paid by member organizations, an infrastructure grant from the Ministère du Développement économique et régional et de la Recherche, and grants and research mandates obtained by its research teams.

\section{Les partenaires du CIRANO}

\section{Partenaire majeur}

Ministère du Développement économique, de l'Innovation et de l'Exportation

\section{Partenaires corporatifs}

Autorité des marchés financiers

Banque de développement du Canada

Banque du Canada

Banque Laurentienne du Canada

Banque Nationale du Canada

Banque Royale du Canada

Banque Scotia

Bell Canada

BMO Groupe financier

Caisse de dépôt et placement du Québec

Fédération des caisses Desjardins du Québec

Financière Sun Life, Québec

Gaz Métro

Hydro-Québec

Industrie Canada

Investissements PSP

Ministère des Finances du Québec

Power Corporation du Canada

Rio Tinto Alcan

State Street Global Advisors

Transat A.T.

Ville de Montréal

\section{Partenaires universitaires}

École Polytechnique de Montréal

HEC Montréal

McGill University

Université Concordia

Université de Montréal

Université de Sherbrooke

Université du Québec

Université du Québec à Montréal

Université Laval

Le CIRANO collabore avec de nombreux centres et chaires de recherche universitaires dont on peut consulter la liste sur son site web.

Les cahiers de la série scientifique (CS) visent à rendre accessibles des résultats de recherche effectuée au CIRANO afin de susciter échanges et commentaires. Ces cahiers sont écrits dans le style des publications scientifiques. Les idées et les opinions émises sont sous l'unique responsabilité des auteurs et ne représentent pas nécessairement les positions du CIRANO ou de ses partenaires.

This paper presents research carried out at CIRANO and aims at encouraging discussion and comment. The observations and viewpoints expressed are the sole responsibility of the authors. They do not necessarily represent positions of CIRANO or its partners. 


\title{
Local Utility and Multivariate Risk Aversion
}

\author{
Arthur Charpentier ${ }^{*}$, Alfred Galichon ${ }^{\dagger}$, Marc Henry ${ }^{*}$
}

\section{Résumé / Abstract}

We revisit Machina's local utility as a tool to analyze attitudes to multivariate risks. Using martingale embedding techniques, we show that for non-expected utility maximizers choosing between multivariate prospects, aversion to multivariate mean preserving increases in risk is equivalent to the concavity of the local utility functions, thereby generalizing Machina's result in [18]. To analyze comparative risk attitudes within the multivariate extension of rank dependent expected utility of [10], we extend Quiggin's monotone mean and utility preserving increases in risk and show that the useful characterization given in [17] still holds in the multivariate case.

Mots clés/Keywords : local utility, multivariate risk aversion, multivariate rank dependent utility, pessimism, multivariate Bickel-Lehmann dispersion.

Codes JEL : D63, D81, C61

\footnotetext{
*. National Institute of Statistics and Economic Studies (INSEE) - National School for Statistical and Economic Administration (ENSAE).

${ }^{\dagger}$ Ecole Polytechnique, Paris - Department of Economic Sciences.

¥CIRANO and Université de Montréal. Adress for correspondence: Département de sciences économiques, Université de Montréal, C.P. 6128, succursale Centre-ville, Montréal, Québec, H3C 3J7, Canada, marc.henry@umontreal.ca.
} 


\section{INTRODUCTION}

One of the many appealing features of expected utility theory is the characterization of attitudes towards risk through the shape of the utility function. Following extensive evidence of violations of the independence axiom which delivers linearity in probabilities of the functional characterizing preferences over risky prospects, most notably the celebrated Allais paradox [1], Machina showed in [18], [19] that smoothness of the preference functional was sufficient to recover representability of risk attitudes through a local approximation, which he called local utility function. Parallel to the study of risk attitudes in generalized expected utility theories, [28] and [16] analyzed attitudes to the combination of income risk and price risk in preferences over multiple commodities within the expected utility framework. This paper is concerned with non expected utility analysis of attitudes to multivariate risks. So far, three approaches have emerged to analyze attitudes to multivariate risks without the independence axiom in [31], [24] and [12]. All three apply dimension reduction devices to preferences over multivariate prospects. [31] considers rank dependent utility over multivariate prospects with stochastically independent components only; [24] show additive separability of the local utility function under a property they call dominance (equivalent to the notion of correlation neutrality in [9]) and [12] show that under a property they call degenerate independence, preferences over uncertain multivariate prospects can be fully recovered from preferences over uncertain income and preferences over deterministic multivariate outcomes. We consider the general case, where attitudes to income risk and price risk cannot be separated in this way and show that in general smooth preferences over multivariate prospects, the main result of [18] still holds, and aversion to increases in risk is equivalent to concavity of the local utility function. The proof relies on the martingale characterization of increasing risk in [10] and martingale embedding theory, specifically [14]. A special case of this result appears in [10], who derive the family of local utility functions in a multivariate rank dependent utility model under aversion to multivariate mean preserving increases in risk. Machina also showed in [18] that interpersonal comparisons of risk aversion can 
be characterized by properties of the local utility function. Karni generalizes in [15] the equivalence between decreasing certainty equivalents and concave transformations of the local utility functions to smooth preferences over multivariate prospects. To complement this result, we extend the notion of compensated spread to multivariate prospects and generalize the characterization of Quiggin's monotone increases in risk [21] as mean preserving comonotonic spreads in [17]. We also generalize Quiggin's notion of pessimism and characterize pessimistic decision functionals by the shape of their local utility function. We apply these notions to interpersonal comparison of risk aversion within the multivariate rank dependent model of [10] and we show that pessimism is equivalent to weak risk aversion in that framework.

The rest of the paper is organized as follows. Section 1 defines local utility. Section 2 shows that aversion to mean preserving increases in risk is equivalent to concavity of the local utility functions and Section 3 extends Quiggin's monotone mean preserving increases in risk and applies it to interpersonal comparisons of risk aversion within the multivariate rank dependent utility model. The last section concludes.

Notation and basic definitions. Let $(S, \mathcal{F}, \mathbb{P})$ be a non-atomic probability space. Let $X: S \rightarrow \mathbb{R}^{d}$ be a random vector. We denote the cumulative distribution function of $X$ by $F_{X} . \mathbb{E}$ is the expectation operator with respect to $\mathbb{P}$. For $x$ and $y$ in $\mathbb{R}^{d}$, let $x \cdot y$ be the standard scalar product of $x$ and $y$, and $\|x\|^{2}$ the Euclidian norm of $x$. We denote by $X={ }_{d} \mu$ the fact that the distribution of $X$ is $\mu$ and by $X={ }_{d} Y$ the fact that $X$ and $Y$ have the same distribution. $Q_{X}$ denotes the quantile function of distribution $X$. In dimension 1 , this is defined for all $t \in[0,1]$ by $Q_{X}(t)=\inf _{x \in \mathbb{R}}\{\operatorname{Pr}(X \leq x)>t\}$. In larger dimensions, it is defined in Definition 5 of Section 3.2 below. We call $L_{d}^{2}$ the set of random vectors $X$ in dimension $d$ such that $\mathbb{E}\|X\|^{2}<\infty$. We denote by $\mathcal{D}$ the subset of $L_{d}^{2}$ containing random vectors with a density relative to Lebesgue measure. A functional $\Phi$ on $L_{d}^{2}$ is called upper semi-continuous (denoted u.s.c.) if for any real number $\alpha,\left\{X \in L_{d}^{2}: \Phi(X)>\alpha\right\}$ is open. A functional $\Phi$ is lower semi-continuous (l.s.c.) if $-\Phi$ is upper semi-continuous. $\Phi$ is called law-invariant if $\Phi(X)=\Phi(\tilde{X})$ whenever $\tilde{X}={ }_{d} X$. By a slight abuse of notation, when $\Phi$ is law invariant, $\Phi\left(F_{X}\right)$ will 
be used to denote $\Phi(X)$. For a convex lower semi-continuous function $V: \mathbb{R}^{d} \mapsto \mathbb{R}$, we denote by $\nabla V$ its gradient (equal to the vector of partial derivatives).

\section{Local Utility}

We consider decision makers choosing among multivariate uncertain prospects $X \in$ $\mathcal{D}$. We assume that the decision makers' preferences over $\mathcal{D}$ are given as a complete, reflexive and transitive binary relation represented by a real valued functional $\Phi$, which is continuous relative to the topology of convergence in distribution. We further assume that $\Phi$ is law-invariant. For a given prospect distribution $F$, if there exists a function $U(x ; F)$ such that

$$
\Phi\left(F^{*}\right)-\Phi(F)-\int U(x ; F)\left[d F^{*}(x)-d F(x)\right] \rightarrow 0
$$

when $F^{*}$ converges to $F$ in distribution, then $U(x ; F)$ is called local utility function relative to $\Phi$ at $F$. Since expected utility preferences are linear in probabilities, the local utility of an expected utility decision maker is constant and equal to her utility function. Theorem 1 in [18] shows that smooth preference functionals are monotonic if and only if their local utility functions are increasing. This can be extended to the case of multivariate prospects.

Definition 1. A prospect $X \in \mathcal{D}$ is said to dominate stochastically a prospect $Y$ (denoted $X \succsim_{S D} Y$ ) if there exist $\tilde{X}={ }_{d} X$ and $\tilde{Y}={ }_{d} Y$ such that $\tilde{X} \geq \tilde{Y}$ almost surely, where $\geq$ denotes componentwise order in $\mathbb{R}^{n}$.

A preference functional is said to preserve stochastic dominance if stochastically dominant prospects are always preferred. If the preference functional $\Phi$ is law invariant and monotonic, in the sense that $\Phi(X) \geq \Phi(Y)$ when $X$ yields larger outcomes than $Y$ in almost all states, then it preserves stochastic dominance. Then we have the rather straightforward multivariate generalization of Theorem 1 of [18] (mentioned without proof in [24]).

Proposition 1 (Monotonicity). Let $\Phi$ be a law invariant preference functional, which admits a local utility $U(x ; F)$ for all $F$. Then the following statements are equivalent. 
(i) $\Phi$ is monotone, i.e., $\Phi(X) \geq \Phi(Y)$ when $X \geq Y$ a.s., (ii) $\Phi$ preserves stochastic dominance and (iii) $U(x ; F)$ is nondecreasing in $x$ for all $F$.

Proof of Proposition 1. Take any two multivariate prospects $X_{1}$ and $X_{0}$ such that $X_{0} \leq X_{1}$ almost surely, where the inequality is component-wise. Define $X_{t}=t X_{1}+$ $(1-t) X_{0}$. A law invariant preference functional is increasing with respect to first order stochastic dominance if and only if it is monotone, i.e., if $\Phi\left(X_{t}\right)$ is a non decreasing function of $t$. Denote by $U_{\Phi}\left(\cdot ; F_{X}\right)$ the local utility function of $\Phi$ at $F_{X}$. We have the following:

$$
\begin{aligned}
\frac{d}{d t} \Phi\left(X_{t}\right) & =\frac{d}{d t} \Phi\left(t X_{1}+(1-t) X_{0}\right) \\
& =\mathbb{E}\left[\nabla U_{\Phi}\left(X_{t}, F_{X_{t}}\right) \cdot \frac{d X_{t}}{d t}\right] \\
& =\mathbb{E}\left[\nabla U_{\Phi}\left(X_{t}, F_{X_{t}}\right) \cdot\left(X_{1}-X_{0}\right)\right]
\end{aligned}
$$

Hence $\Phi$ is monotone if and only if $\nabla U_{\Phi}\left(\cdot ; F_{X}\right) \geq 0$ for all $F_{X}$, which completes the proof.

If in addition, the decision maker is indifferent to correlation increasing transfers, or correlation neutral according to the terminology of [9], then Safra and Segal show in [24] that the local utility functions are additively separable, namely that $U(x ; F)=$ $\sum_{j=1}^{n} U_{j}\left(x_{j} ; F\right)$, where $x_{j}$ is the $j$-th component of the outcome $x \in \mathbb{R}^{n}$. Yaari's rank dependent utility maximizers over stochastically independent $d$-dimensional risks in [31] are represented by

$$
\Phi(X)=\sum_{i=1}^{d} \alpha_{i} \int_{0}^{1} \phi_{i}(u) Q_{X_{i}}(t) d t,
$$

where $Q_{X_{i}}$ is the quantile function of component $X_{i}$ of the risk $X$, the $\phi_{i}{ }^{\prime}$ s, $i=1, \ldots, d$, are non-negative functions on $[0,1]$ (quantile weights interpreted as probability distortions) and the $\alpha_{i}$ 's, $i=1, \ldots, d$, are positive weights. The local utility of decision maker $\Phi$ is given by

$$
U(x ; F)=\sum_{i=1}^{d} \alpha_{i} \int^{x_{i}} \phi_{i}\left(F_{i}(z)\right) d z
$$


where $F_{i}$ is the $i$-th marginal of distribution $F$ (see for instance Section 4 of [26]).

\section{RISK AVERSION}

We now show that attitude to risk with smooth preference over multivariate prospects can be characterized by the shape of local utilities, as was proved in the case of univariate risks in Theorem 2 of [18]. The latter shows that aversion to mean preserving increases in risk is equivalent to concavity of local utility functions. Extending this result to preferences over multivariate prospects calls for a generalization of the notion of mean preserving increase in risk proposed in [23].

Definition 2 (Mean preserving increase in risk). A prospect $Y \in \mathcal{D}$ is called a mean preserving increase in risk (hereafter MPIR) of a prospect $X \in \mathcal{D}$, denoted $X \succsim_{M P I R}$ $Y$, if any of the following equivalent statements hold.

(a) For all bounded concave functions $f$ on $\mathbb{R}^{n}, \mathbb{E} f(X) \geq \mathbb{E} f(Y)$.

(b) There exists $\tilde{Y}={ }_{d} Y$ such that $(X, \tilde{Y})$ is a martingale, i.e., $\mathbb{E}[\tilde{Y} \mid X]=X$.

(c) For all u.s.c. law invariant concave functionals $\Psi$ on $\mathcal{D}, \Psi(X) \geq \Psi(Y)$.

The equivalence between (a) and (b) is due to [29] and the interpretation as an increase in risk is the same as in [23] for the univariate case. An immediate corollary of (c) (shown to be equivalent to (a) and (b) in [10]) is that cardinal risk aversion, i.e., concavity of the functional $\Phi$ representing preferences, implies ordinal risk aversion, in the sense of aversion to mean preserving increases in risk. We can now state the main result of this section, which is a direct generalization of Theorems 2 and 3 of [18].

Theorem 1 (Risk aversion and local utility). Let $\Phi$ be a law invariant preference functional, which admits a local utility $U(x ; F)$ for all $F$ in $\mathcal{D}$. Then the following statements are equivalent. (i) $\Phi$ is risk averse, i.e., $\Phi(X) \geq \Phi(Y)$ when $Y$ is an MPIR of $X$, (ii) $U(x ; F)$ is a concave function of $x$ for all $F$ and (iii) For arbitrary distributions $F$ and $F^{* *}$ in $\mathcal{D}$ and arbitrary probability $p, \Phi\left((1-p) F^{* *}+p G_{\mu_{F}}\right) \geq$ 
$\Phi\left((1-p) F^{* *}+p F\right)$, where $\mu_{F}$ is the mean of $F$ and $G_{\mu}$ is the degenerate distribution at $\mu$.

Proof of Theorem 1. In the following, we use the two alternative notations, $\Phi(X)=$ $\Phi(F)$ where $X={ }_{d} F$, and the equivalent expression of the local utility equivalently, using the equivalence between the Gâteaux and the Fréchet derivative, when the latter exists: for all $H$,

$$
U(\cdot, F)=\left.\frac{\partial}{\partial \varepsilon} \Phi((1-\varepsilon) F+\varepsilon H)\right|_{\varepsilon=0}
$$

We denote $F^{*}=(1-\varepsilon) F+\varepsilon H$.

(ii) $\Longrightarrow($ i): Suppose that $x \mapsto U(x ; F)$ is a concave function, for any distribution $F$. Let $X$ be a random variable with distribution $F$, and consider $Y$ such that $Y$ is a MPIR of $X$, i.e., there exists $\tilde{Y}={ }_{d} Y$ such that $(X, \tilde{Y})$ is a martingale, i.e., $\mathbb{E}[\tilde{Y} \mid X]=X$. Then, following [14], there exists a continuous martingale $\left(\tilde{Y}_{t}, t \in[0,1]\right)$ such that $\tilde{Y}_{1}=\tilde{Y}$ and $\tilde{Y}_{0}=X$. More precisely, from Corollary 4.1.25 and Proposition 5.3.2 in [22], there exists $\left(\sigma_{t}\right)$ such that $d \tilde{Y}_{t}=\sigma_{t} d B_{t}$ where $\left(B_{t}\right)$ is a standard $d$ dimensional Brownian motion, and $\sigma_{t}=\left[\sigma_{i, j, t}\right]_{i, j=1, \cdots, d}$. Let $F_{t}$ denote the distribution of $\tilde{Y}_{t}$. From (i), since $\left(\tilde{Y}_{t}, t \in[0,1]\right)$ is a martingale, $\Phi\left(\tilde{Y}_{s}\right) \geq \Phi\left(\tilde{Y}_{t}\right)$ if $0 \leq s \leq t \leq 1$, or equivalently $t \mapsto \Phi\left(F_{t}\right)$ is decreasing. Further, if $d t>0$,

$$
\Phi\left(F_{t+d t}\right)-\Phi\left(F_{t}\right)=\mathbb{E}\left(U\left(\tilde{Y}_{t+d t} ; F_{t+d t}\right)-U\left(\tilde{Y}_{t} ; F_{t}\right)\right)
$$

i.e.,

$\Phi\left(F_{t+d t}\right)-\Phi\left(F_{t}\right)=\mathbb{E}\left(\left[U\left(\tilde{Y}_{t+d t} ; F_{t+d t}\right)-U\left(\tilde{Y}_{t+d t} ; F_{t}\right)\right]+\left[U\left(\tilde{Y}_{t+d t} ; F_{t}\right)-U\left(\tilde{Y}_{t} ; F_{t}\right)\right]\right)$

For the second part, from Itô's Lemma,

$U\left(\tilde{Y}_{t+d t} ; F_{t}\right)-U\left(\tilde{Y}_{t} ; F_{t}\right)=\int_{t}^{t+d t} \sum_{i=1}^{d} \frac{\partial U\left(\tilde{Y}_{s} ; F_{t}\right)}{\partial y_{i}} d \tilde{Y}_{s}^{i}+\frac{1}{2} \sum_{i, j=1}^{d} \int_{t}^{t+d t} \frac{\partial^{2} U\left(\tilde{Y}_{s} ; F_{t}\right)}{\partial y_{i} y_{j}} d<\tilde{Y}_{s}^{i}, \tilde{Y}_{s}^{j}>$ 
where $d \tilde{Y}_{s}^{i}=\sum_{j=1}^{d} \sigma_{i, j, s} d B_{s}^{j}$, while $d<\tilde{Y}_{s}^{i}, \tilde{Y}_{s}^{j}>=\sigma_{s}^{i \prime} \delta_{i, j} \sigma_{s}^{j} d s$. If we take the expected value, the first part is zero, and therefore

$$
\mathbb{E}\left[U\left(X_{t+d t} ; F_{t}\right)-U\left(X_{t} ; F_{t}\right)\right]=\frac{1}{2} \sum_{i, j=1}^{d} \int_{t}^{t+d t} \frac{\partial^{2} U\left(\tilde{Y}_{s} ; F_{t}\right)}{\partial y_{i} y_{j}} d \sigma_{s}^{i \prime} \delta_{i, j} \sigma_{s}^{j} d s
$$

Thus, if $d t \downarrow 0$,

$$
\mathbb{E}\left[U\left(X_{t+d t} ; F_{t}\right)-U\left(X_{t} ; F_{t}\right)\right]=\frac{1}{2} \mathbb{E}\left[\operatorname{trace}\left(D^{2} U\left(X_{t} ; F_{t}\right)\right) \sigma_{t} \sigma_{t}^{*}\right] d t
$$

and since $x \mapsto U(x ; F)$ is a concave function, it follows that for all $t, \Phi\left(F_{t+d t}\right)-\Phi\left(F_{t}\right) \leq$ 0, i.e. $t \mapsto \Phi\left(F_{t}\right)$ is decreasing. And therefore $\Phi\left(F_{1}\right) \leq \Phi\left(F_{0}\right)$, or equivalently $\Phi(Y)=\Phi(\tilde{Y}) \leq \Phi(X)$.

(i) $\Longrightarrow$ (ii): let $Y$ be MPIR of $X$, so that there exists $\tilde{Y}={ }_{d} Y$ such that $(X, \tilde{Y})$ is a martingale, i.e., $\mathbb{E}[\tilde{Y} \mid X]=X$ (from Definition 2). As before, it is possible to interpolate from $X$ to $\tilde{Y}$ with a continuous martingale $\left(\tilde{Y}_{t}\right)$ on $[0,1]$ with increments $d \tilde{Y}_{t}=\sigma_{t} d B_{t}$. Using the same expressions we used earlier, we can derive that

$$
\Phi\left(F_{t+d t}\right)-\Phi\left(F_{t}\right)=\frac{1}{2} \mathbb{E}\left[\operatorname{trace}\left(D^{2} U\left(X_{t} ; F_{t}\right)\right) \sigma_{t} \sigma_{t}^{*}\right] d t
$$

Hence, since $t \mapsto \Phi\left(F_{t}\right)$ is decreasing, it means that $\mathbb{E}\left[\operatorname{trace}\left(D^{2} U\left(X_{t} ; F_{t}\right)\right) \sigma_{t} \sigma_{t}^{*}\right] \leq 0$. It follows that $D^{2} U\left(X_{t} ; F_{t}\right)$ is a symmetric non-positive matrix, and so, $x \mapsto U\left(x ; F_{t}\right)$ is a concave function for all $F_{t}$. In particular for $t=0$.

(i) $\Leftrightarrow$ (iii): Two distributions $G$ and $H$ in $\mathcal{D}$ satisfy $G=(1-p) F^{* *}+p G_{\mu_{F}}$ and $H=(1-p) F^{* *}+p F$ for arbitrary distribution $F^{* *}$ if and only if for each $x$, $H(x)-G(x)=p\left(L^{\mu_{F}}(x)-1\left\{x \geq \mu_{F}\right\}\right)$, for some distribution $L^{\mu_{F}}$ centered at $\mu_{F}$. Call mixture ordering the binary relation so defined between $G$ and $H$. By (b) of Definition 2, $H \succsim_{M P I R} G$ if and only if there exist $Y={ }_{d} H$ and $X={ }_{d} G$ such that $\mathbb{E}[Y \mid X]=X$. So for each $z, H(z)-G(z)=\int\left(F_{Y \mid X}(z \mid \mu)-1\{z \geq \mu\}\right) d G(\mu)$ with $F_{Y \mid X}(z \mid \mu)$ centered at $x$. Hence, the transitive closure of the mixture ordering is the ordering of mean preserving increases in risk and the equivalence between (i) and (iii) follows. 
Using the local utility, we can define a full insurance premium for preferences over multivariate prospects. Let $X \in \mathcal{D}$ be a prospect evaluated by a decision maker with smooth preferences as $\Phi(X)$. A full insurance premium can be defined as an element of the set of vectors $\pi \in \mathbb{R}^{d}$ satisfying $\Phi(X)=U\left(\mathbb{E} X-\pi ; F_{X}\right)$, where $F_{X}$ is the distribution function of the random vector $X$.

\section{INCREASING RISK AVERSION IN MULTIVARIATE RANK DEPENDENT UTILITY}

3.1. Aversion to monotone mean preserving increases in risk. In [21], Quiggin shows that the notion of mean preserving increases in risk is too weak to coherently order rank dependent utility maximizers according to increasing risk aversion. [21] shows that the notion of monotone mean preserving increases in risk (Monotone MPIR) is the weakest stochastic ordering that achieves a coherent ranking of risk aversion in the rank dependent utility framework. Monotone MPIR is the mean preserving version of Bickel-Lehmann dispersion ([2],[3]), which we now define.

Definition 3 (Bickel-Lehmann Dispersion and Monotone Mean Preserving Increase in Risk). Let $Q_{X}$ and $Q_{Y}$ be the quantile functions of the random variables $X$ and $Y$. $X$ is said to be Bickel-Lehmann less dispersed, denoted $X \succsim_{B L} Y$, if $Q_{Y}(u)-Q_{X}(u)$ is a nondecreasing function of $u$ on $(0,1)$. The mean preserving version is called monotone mean preserving increase in risk (hereafter MMPIR) and denoted $\precsim_{M M P I R}$.

MMPIR is a stronger ordering than MPIR in the sense that $X \succsim_{M M P I R} Y$ implies $X \succsim_{M P I R} Y$ since it is shown in [7] that an MPIR can be obtained as the limit of a sequence of simple mean preserving spreads $Y$ of $X$, defined by $Q_{Y}(u)-Q_{X}(u)$ non-positive below some $u_{0} \in[0,1]$ and non-negative above $u_{0}$. [21] relates MMPIR aversion of a rank dependent utility decision maker to a notion he calls pessimism. Aversion to MMPIR is defined in the usual way as follows.

Definition 4. A preference functional $\Phi$ over random prospects is called averse to monotone mean preserving increases in risk if and only if $X \precsim_{M M P I R} Y$ implies $\Phi(X) \geq \Phi(Y)$. 
Consider a decision maker with preference relation characterized by the functional defined for each prospect $X$ by

$$
\Phi(X)=\int_{-\infty}^{\infty} f\left(1-F_{X}(x)\right) d x
$$

with $f(0)=0, f(1)=1$ and $f$ non decreasing. Then Theorem 3 of [6] shows that aversion to MMPIR is equivalent to $f(u) \leq u$ for each $u \in[0,1]$. Since the local utility associated with $\Phi$ is $x \mapsto U_{\Phi}\left(x ; F_{X}\right)=\int^{x} f^{\prime}\left(1-F_{X}(z)\right) d z$, aversion to MMPIR can be characterized with the local utility. We now generalize this local utility characterization of MMPIR aversion beyond rank dependent utility functionals to all preference functionals that admit a local utility.

Theorem 2 (Local utility of MMPIR averse decision makers). Let $\Phi$ be a preference functional with local utility at $X$ denoted $x \mapsto U_{\Phi}\left(x ; F_{X}\right) . \Phi$ is MMPIR averse if and only if

$$
\mathbb{E}\left[\frac{U^{\prime}\left(X ; F_{X}\right)}{\mathbb{E}\left[U^{\prime}\left(X ; F_{X}\right)\right]} 1\{X>x\}\right] \leq \mathbb{E}[1\{X>x\}]
$$

for all $X \in L^{2}$, almost all $x \in \mathbb{R}$.

Remark 1. Note that in the special case of rank dependent utility functional (3.1), the characterization above is equivalent to $f\left(1-F_{X}(x)\right) \leq 1-F_{X}(x)$ for all $x$ and $X$, which is equivalent to $f(u) \leq u$ for all $u \in[0,1]$ as mentioned previously.

In Proposition 2 of [17], Landsberger and Meilijson give a characterization of BickelLehmann dispersion in the spirit of the characterization of MPIR given in the equivalence between (a) and (b) of Proposition 2. In the latter, MPIR increases are characterized by the addition of noise, whereas in the former MMPIR are characterized by the addition of a zero mean comonotonic variable.

Proposition 2 (Landsberger-Meilijson). A random variable $X$ has Bickel-Lehmann less dispersed distribution than a random variable $Y$ if and only iff there exists $Z$ comonotonic with $X$ such that $Y={ }_{d} X+Z$.

Using Proposition 2, we can prove Theorem 2. 
Proof of Theorem 2. From Proposition 2, $\Phi$ is MMPIR averse if and only if $\Phi(X+$ $Z)-\Phi(X) \leq 0$ for any $(X, Z)$ comonotonic and $\mathbb{E} Z=0$. Now for $Z$ small enough,

$$
\begin{aligned}
\Phi(X+Z)-\Phi(X) & =\int_{0}^{1} U_{\Phi}^{\prime}\left(Q_{X}(u) ; F_{X}\right)\left[Q_{X+Z}(u)-Q_{X}(u)\right] d u \\
& =\int_{0}^{1} U_{\Phi}^{\prime}\left(Q_{X}(u) ; F_{X}\right) Q_{Z}(u) d u
\end{aligned}
$$

since the quantile function is comonotonic additive. Therefore we have

$$
\int_{0}^{1} U_{\Phi}^{\prime}\left(Q_{X}(u) ; F_{X}\right) Q_{Z}(u) d u \leq 0
$$

for any $Z$ with mean zero. After changing variables, this yields

$$
\int_{-\infty}^{\infty} U_{\Phi}^{\prime}\left(y ; F_{X}\right) \Delta(y) d F_{X}(y) \leq 0
$$

for any function $\Delta$ increasing and such that $\int_{-\infty}^{\infty} \Delta(y) d F_{X}(y)=0$. Choosing $\Delta(y)=$ $1\{y>x\}-\left[1-F_{X}(x)\right]$ yields the result.

We now show how this notion of Bickel-Lehmann dispersion and the LandsbergerMeilijson characterization can be extended to multivariate prospects and how it can be applied to the ranking of risk aversion of multivariate rank dependent utility maximizers. To that end, we appeal to the multivariate notions of quantiles and comonotonicity developed in [10], [8] and [20].

3.2. Multivariate quantiles and comonotonicity. [10] and [8] define multivariate quantiles by extending the variational characterization of univariate quantiles based on rearrangement inequalities of Hardy, Littlewood and Pólya [13]. The following well known equality

$$
\int_{0}^{1} Q_{X}(u) u d u=\max \{\mathbb{E}[X \tilde{U}]: \tilde{U} \text { uniformly distributed on }[0,1]\},
$$

is extended to the multivariate case to define the quantile $Q_{X}$ of a random vector $X \in$ $\mathcal{D}$ with the the following, where $\mu$ is a reference absolutely continuous distribution on $\mathbb{R}^{d}$ with finite second moment.

$$
\mathbb{E}\left[Q_{X}(U) \cdot U\right]=\max \left\{\mathbb{E}[X \cdot \tilde{U}]: \tilde{U}=_{d} \mu\right\} .
$$


It follows from the theory of optimal transportation (see Theorem 2.12(ii), p. 66 of [30]) that there exists an essentially unique convex lower semi-continuous function $V: \mathbb{R}^{d} \rightarrow \mathbb{R}$ such that $Q_{X}=\nabla V$ satisfies Equation 3.3. Hence the definition of multivariate quantiles due to [10] and [8].

Definition 5 ( $\mu$-quantile). The $\mu$-quantile function of a random vector $X$ in $\mathcal{D}$ with respect to an absolutely continuous distribution $\mu$ on $\mathbb{R}^{d}$ is defined by $Q_{X}$ in Equation 3.3.

This concept of a multivariate quantile is the counterpart of the definition of multivariate comonotonicity in [10] and [8], motivated by the fact that two univariate prospects $X$ and $Y$ are comonotonic if there is a prospect $U$ and non-decreasing maps $T_{X}$ and $T_{Y}$ such that $Y=T_{Y}(U)$ and $X=T_{X}(U)$ almost surely or, equivalently, $\mathbb{E}[U X]=\max \left\{\mathbb{E}[\tilde{U} X]: \tilde{U}={ }_{d} U\right\}$ and $\mathbb{E}[U Y]=\max \left\{\mathbb{E}[\tilde{U} Y]: \tilde{U}={ }_{d} U\right\}$.

Definition 6 ( $\mu$-comonotonicity). Random vectors $X$ and $Y$ in $\mathcal{D}$ are called $\mu$ comonotonic if there exists $U={ }_{d} \mu$ such that $\mathbb{E}[X \cdot U]=\max \left\{\mathbb{E}[\tilde{X} \cdot U]: \tilde{X}={ }_{d} X\right\}$ and $\mathbb{E}[Y \cdot U]=\max \left\{\mathbb{E}[\tilde{Y} \cdot U]: \tilde{Y}={ }_{d} Y\right\}$.

Two random vectors are $\mu$-comonotonic if they can be rearranged simultaneously so that they are both equal to their $\mu$-quantile. Another variational notion of multivariate comonotonicity, called $c$-comonotonicity, is proposed in [20].

Definition 7 (c-comonotonicity). Random vectors $X$ and $Y$ in $\mathcal{D}$ are called $c$ comonotonic if there exists a convex function $V$ such that $Y=\nabla V(X)$.

Both $\mu$-comonotonicity and $c$-comonotonicity will feature in the extension of BickelLehmann dispersion in the following section.

3.3. Multivariate Bickel-Lehmann dispersion. The Bickel-Lehmann dispersion order and its mean-preserving version in [21], monotone MPIR, rely on the notion of monotone single crossings, hence on the monotonicity of the function $Q_{Y}-Q_{X}$. A natural extension of the class of non-decreasing functions to functions on $\mathbb{R}^{d}$ is the 
class of gradients of convex functions, whose definition doesn't rely on the ordering on the real line. Hence the following definition of $\mu$-Bickel-Lehmann dispersion, which depends on the baseline distribution $\mu$ relative to which multivariate quantiles are defined.

Definition 8 ( $\mu$-Bickel-Lehmann dispersion). A random vector $X \in \mathcal{D}$ is called $\mu$ Bickel-Lehmann less dispersed than a random vector $Y \in \mathcal{D}$, denoted $X \succsim_{\mu B L} Y$, if there exists a convex function $V: \mathbb{R}^{d} \rightarrow \mathbb{R}$ such that the $\mu$-quantiles $Q_{X}$ and $Q_{Y}$ of $X$ and $Y$ satisfy $Q_{Y}(u)-Q_{X}(u)=\nabla V(u)$ for $\mu$-almost all $u \in[0,1]^{d}$.

As defined above, $\mu$-Bickel-Lehmann dispersion defines a transitive binary relation, and therefore an order on $\mathcal{D}$. Indeed, if $X \succsim_{\mu B L} Y$ and $Y \succsim_{\mu B L} Z$, then $Q_{Y}(u)-$ $Q_{X}(u)=\nabla V(u)$ and $Q_{Z}(u)-Q_{Y}(u)=\nabla W(u)$. Therefore, $Q_{Z}(u)-Q_{X}(u)=$ $\nabla(V(u)+W(u))$ so that $X \succsim_{\mu B L} Z$. When $d=1$, this definition simplifies to definition 3 .

3.3.1. Characterization. We have the following generalization of the LandsbergerMeilijson characterization of Proposition 2.

Theorem 3. A random vector $X \in \mathcal{D}$ is $\mu$-Bickel-Lehmann less dispersed than a random vector $Y \in \mathcal{D}$ if and only if there exists a random vector $Z \in \mathcal{D}$ such that (i) $X$ and $Z$ are $\mu$-comonotonic and (ii) $Y={ }_{d} X+Z$.

Proof of Theorem 3. Assume $X \succsim_{\mu B L} Y$ and call $Q_{X}$ and $Q_{Y}$ the $\mu$-quantiles of $X$ and $Y$. Let $U$ be a random vector with distribution $\mu$ such that $X=Q_{X}(U)$. By assumption, $\nabla V(U)$ is equal to $Q_{Y}(U)-Q_{X}(U)=Q_{Y}(U)-X$. Call $Z=\nabla V(U)$. By Theorem 2.12(ii), p. 66 of [30], $\nabla V$ is the $\mu$-quantile $Q_{Z}$ of $Z$. Hence we have $X=Q_{X}(U)$ and $Z=Q_{Z}(U)$ and $X$ and $Z$ are therefore $\mu$-comonotonic and we have $Y={ }_{d} Q_{Y}(U)=X+Z$ as required. Conversely, take $X$ and $Z \mu$-comonotonic. Then $X=Q_{X}(U)$ and $Z=Q_{Z}(U)$ for some $U={ }_{d} \mu$, where $Q_{X}$ and $Q_{Z}$ are the $\mu$-quantiles of $X$ and $Z$ respectively. Call $Y=X+Z$ and $Q_{Y}=Q_{X+Z}$ the $\mu$-quantile of $Y$. In the proof of Theorem 1 of [10], it is shown that $Q_{X+Z}=Q_{X}+Q_{Z}$ when $X$ and $Z$ 
are $\mu$-comonotonic. Hence, we have $Q_{Y}=Q_{X}+Q_{Z}$, i.e., $Q_{Y}-Q_{X}=Q_{Z}$, and $Q_{Z}$ is the gradient of a convex function by Definition 5 . The result follows.

The characterization given in Theorem 3 now allows us to generalize our characterization of MMPIR aversion to the multivariate case.

Proposition 3 (Local utility of multivariate MMPIR averse decision makers). A decision functional $\Phi$ is $\mu-M M P I R$ averse if and only if its local utility function satisfies

$$
\mathbb{E}_{\mu}\left[\nabla V(U) \cdot \nabla U_{\Phi}\left(\nabla V_{X}(U) ; F_{X}\right)\right] \leq 0
$$

for all $V$ convex with $\mathbb{E}_{\mu} V(U)=0$.

Proof of Proposition 3. Let $Y$ dominate $X$ with respect to mean preserving $\mu$-BickelLehmann dispersion, i.e., $Y \succsim_{\mu-M M P I R} X$. This is equivalent to $Y={ }_{d} X+Z$ with $X$ and $Z \mu$-comonotonic, $\mathbb{E} Z=0$. For each $\epsilon>0$, define $Y_{\epsilon}=X+\epsilon Z$, which also dominates $X$ with respect to $\mu$-Bickel-Lehmann dispersion. $\Phi$ is $\mu$ MMPIR averse if and only if for all $\epsilon>0, \Phi(X+\epsilon Z)-\Phi(X) \leq 0$. Denoting $Q_{X+\epsilon Z}$ and $Q_{X}$ the $\mu$-quantiles of $Y_{\epsilon}$ and $X$ respectively and $U={ }_{d} \mu$, comonotonicity of $X$ and $Z$ implies $Q_{Y_{\epsilon}}(U)=Q_{X+\epsilon Z}(U)=Q_{X}(U)+\epsilon Q_{Z}(U)$. Hence, $0 \geq \Phi(X+\epsilon Z)-\Phi(X)=\mathbb{E} U_{\Phi}\left(X+\epsilon Z ; F_{X}\right)-\mathbb{E} U_{\Phi}\left(X ; F_{X}\right)=\mathbb{E} U_{\Phi}\left(Q_{X+\epsilon Z}(U) ; F_{X}\right)-$ $\mathbb{E} U_{\Phi}\left(Q_{X}(U) ; F_{X}\right)=\mathbb{E} U_{\Phi}\left(Q_{X}(U)+\epsilon Q_{Z}(U) ; F_{X}\right)-\mathbb{E} U_{\Phi}\left(Q_{X}(U) ; F_{X}\right)=\mathbb{E}\left[\epsilon Q_{Z}(U) \cdot\right.$ $\left.\nabla U_{\Phi}\left(Q_{X}(U) ; F_{X}\right)\right]+o(\epsilon)$. Hence, $\mathbb{E}\left[\epsilon Q_{Z}(U) \cdot \nabla U_{\Phi}\left(Q_{X}(U) ; F_{X}\right)\right] \leq 0$, which completes the proof.

The characterization given in Theorem 3 is also crucial to the results in the next section on comparative risk attitudes of multivariate rank dependent utility maximizers.

3.3.2. Relation to other multivariate dispersion orders. We now look at the relation between $\mu$-Bickel-Lehmann dispersion and other generalizations of Bickel-Lehmann dispersion proposed in the statistical literature. The notion of strong dispersion was proposed by [11]. 
Definition 9 (Strong dispersive order). $Y$ is said to dominate $X$ in the strong dispersive order, denoted $Y \succsim_{S D} X$ if $Y={ }_{d} \phi(X)$, where $\phi$ is an expansion, i.e., such that $\left\|\phi(x)-\phi\left(x^{\prime}\right)\right\| \geq\left\|x-x^{\prime}\right\|$ for all pairs $\left(x, x^{\prime}\right)$.

The following Proposition gives conditions under which $\mu$-Bickel-Lehmann implies [11]'s strong dispersion.

Proposition 4. Let $X$ and $Y$ be two random vectors in $\mathcal{D}$. The following propositions hold.

1. $Y$ is more dispersed than $X$ in the strong dispersion order, i.e., $Y \succsim_{S D} X$, if $Y={ }_{d} X+Z$, where $X$ and $Z$ are c-comonotonic.

2. If $Y \succsim_{\mu B L} X$ and the $\mu$-quantiles of $X$ and $Y$ are gradients of strictly convex functions, then $Y \succsim_{S D} X$.

Proof of Proposition 4. If $Y \succsim_{\mu-B L} X$, then by Theorem 3, $Y={ }_{d} X+Z$, where $X$ and $Z$ are $\mu$-comonotonic. Hence

$$
Y={ }_{d} Q_{X+Z}(U)=Q_{X}(U)+Q_{Z}(U)={ }_{d} X+Q_{Z}\left(Q_{X}^{-1}(X)\right),
$$

where $Q_{X}=\nabla V_{X}$ and $Q_{Z}=\nabla V_{Z}$ are gradients of convex functions. Therefore, denoting $\phi(x)=x+\psi(x)=x+\nabla V_{Z} \circ\left(\nabla V_{X}\right)^{-1}(x)$, we need to show that $\phi$ satisfies the $J_{\phi}^{T}(x) J_{\phi}(x)-I \geq 0$ for all $x$ as in the characterization of the strong dispersive order in Theorem 2 of [11]. This follows from the fact that the jacobian of a gradient of a strictly convex function is symmetric positive definite and hence that

$$
J_{\psi}(x)=\left[J_{\nabla V_{X}}\left(\left(\nabla V_{X}\right)^{-1}(x)\right)\right]^{-1}\left[J_{\nabla V_{Z}}\left(\left(\nabla V_{X}\right)^{-1}(x)\right)\right]
$$

is diagonalizable with positive eigenvalues. This completes the proof of (ii). The proof of (i) follows the same lines with $Y={ }_{d} X+Q_{Z}(X)$, where $Q_{Z}$ is the gradient of a convex function.

\subsection{Increasing risk aversion and multivariate rank dependent utility. To} make interpersonal comparisons of attitudes to multivariate risk, we define compensated increases in risk in the spirit of [7]. 
Definition 10 (Compensated Increases in Risk). Let $\Phi$ be the functional representing a decision maker's preferences over multivariate prospects in $\mathcal{D}$. A prospect $Y \in \mathcal{D}$ is a compensated increase in risk from the point of view of $\Phi$ if $X \succsim_{\mu B L} Y$ and $\Phi(Y)=\Phi(X)$.

A ranking of risk aversion is then derived in the usual way, except that the ranking of aversion to multivariate risks is predicated on the reference measure $\mu$ in the definition of dispersion.

Definition 11 (Increasing risk aversion). A decision maker $\tilde{\Phi}$ is more risk averse than a decision maker $\Phi$ if $\tilde{\Phi}$ is averse to a compensated increase in risk from the point of view of $\Phi$, i.e., if $X \succsim_{\mu B L} Y$ and $\Phi(Y)=\Phi(X)$ imply $\tilde{\Phi}(Y) \leq \tilde{\Phi}(X)$.

In the special case of rank dependent utility maximizers, aversion to monotone MPIR and increasing risk aversion take a very simple form. We consider here the multivariate generalization of Yaari decision makers given in [10]. A multivariate rank dependent utility maximizer is characterized by a functional $\Phi$ on multivariate prospects $X \in \mathcal{D}$, which is a weighted sum of $\mu$-quantiles, i.e.,

$$
\Phi(X)=\mathbb{E}\left[Q_{X}(U) \cdot \phi(U)\right],
$$

where $Q_{X}$ is the $\mu$-quantile of $X, U={ }_{d} \mu$ and $\phi(U) \in \mathcal{D}$. As shown in Theorem 1 of [10], $\Phi(X+Z)=\Phi(X)+\Phi(Z)$ when $X$ and $Z$ are $\mu$-comonotonic. Hence we immediately find the following characterization of monotone MPIR aversion and increasing risk aversion.

Theorem 4 (Rank dependent utility). Let $\Phi$ and $\tilde{\Phi}$ be multivariate rank dependent utility functionals, i.e., $\Phi$ and $\tilde{\Phi}$ satisfy (3.4). Then the following hold.

(a) $\Phi$ is averse to a monotone MPIR (i.e., a mean preserving $\mu$-Bickel-Lehmann dispersion) if and only if for all $Z \in \mathcal{D}, \Phi(Z) \leq \Phi(\mathbb{E} Z)$.

(b) $\tilde{\Phi}$ is more risk averse than $\Phi$ iff for all $Z \in \mathcal{D}, \Phi(Z)=0 \Rightarrow \tilde{\Phi}(Z) \leq 0$.

It turns out, therefore, that aversion to MMPIR in the multivariate rank dependent utility model is equivalent to weak risk aversion $(\mathbb{E} X$ preferred to $X)$. Since Theorem 2 
of [10] shows that aversion to MPIR in the multivariate RDU model is equivalent to $\phi(u)=-\alpha u+u_{0}$, with $\alpha>0$ and $u_{0} \in \mathbb{R}^{d}$, we recover the fact that MPIR averters are also monotone MPIR averters as in the univariate case.

Corollary 1. If $\Phi$ is averse to mean preserving increases in risk, than it is also averse to monotone mean preserving increases in risk.

Yaari's rank dependent utility maximizers over stochastically independent multivariate risks in [31] are special cases of (3.4) where the reference distribution $\mu$ has independent marginals. In that special case, (a) of Theorem 4 is equivalent to concavity of the local utility function in (1.2) (i.e., non-increasing $\phi_{i}$ for each $i$ ) and (b) of Theorem 4 is equivalent to $\tilde{\phi}_{i}$ being a decreasing transformation of $\phi_{i}$ for each $i$, so that we recover the classical results of [31].

\section{Conclusion}

Attitudes to multivariate risks were characterized using Machina's local utility in a framework, where objects of choice are multidimensional prospects. Aversion to mean preserving increases in multivariate risk is characterized by concavity of the local utility function as in the univariate case. Comparative attitudes are characterized within the multivariate extension in [10] of rank dependent utility with the help of a multivariate extension of Quiggin's monotone mean preserving increase in risk notion and a generalization of its characterization in [17]. Characterization and derivation of risk premia within the multivariate rank dependent utility model is the natural next step in this research agenda.

\section{REFERENCES}

[1] Allais M., "Le comportement de l'homme rationnel devant le risque: Critiques des postulats et axiomes de l'école américaine," Econometrica 21, pp. 503-546, 1953.

[2] Bickel, P. and E. Lehmann, "Descriptive statistics for non-parametric models, III: Dispersion," Annals of Statistics 4, 11391158, 1976.

[3] Bickel, P. and E. Lehmann, "Descriptive statistics for non-parametric models, IV: Spread," in: Jureckova, J. (ed.) Contributions to statistics. Boston, MA: Reidel, 1979. 
[4] Carlier, G., R.-A. Dana and A. Galichon, "Pareto efficiency for the concave order and multivariate comonotonicity," Journal of Economic Theory, forthcoming.

[5] Carlier, G., A. Galichon and L. Santambrogio, "From Knothe's transport to Brenier's map and a continuation method for optimal transport," SIAM Journal of Mathematical Analysis 41, pp. 2554-2576, 2010.

[6] Chateauneuf, A., M. Cohen and I. Meilijson, "Four notions of mean-preserving increase in risk, risk attitudes and applications to the rank-dependent expected utility model," Journal of Mathematical Economics 40, pp. 547-571, 2004.

[7] Diamond, P. and J. Stiglitz, "Increases in risk and in risk aversion," Journal of Economic Theory 8, pp. 337-360, 1974.

[8] Ekeland, I., A. Galichon and M. Henry, "Comonotone measures of multivariate risks," Mathematical Finance, forthcoming.

[9] Epstein, L. and S. Tanny, "Increasing generalized correlation: a definition and some economic consequences," Canadian Journal of Economics 13, pp. 16-34, 1980.

[10] Galichon, A. and M. Henry, "Dual theory of choice with multivariate risks," Journal of Economic Theory forthcoming.

[11] Giovagnoli, A. and H. Wynn, "Multivariate dispersion orderings," Statistics and Probability Letters 22, pp. 325-332, 1995.

[12] Grant, S., A. Kajii and B. Polak, "Many good choice axioms: when can many-good lotteries be treated as money lotteries," Journal of Economic Theory 56, pp. 313-337, 1992.

[13] Hardy, G., J. Littlewood and G. Pólya, Inequalities. Cambridge: Cambridge University Press, 1952.

[14] Heath, D., "Interpolation of martingales," Annals of Probability 5, pp. 804-806, 1977.

[15] Karni, E., "Generalized expected utility analysis of multivariate risk aversion," International Economic Review 30, pp. 297-305, 1989.

[16] Kihlstrom, R. and L. Mirman, "Risk aversion with many commodities," Journal of Economic Theory 8, pp. 361-368, 1974.

[17] M. Landsberger and I. Meilijson, "Comonotone allocations, Bickel-Lehmann dispersion and the Arrow-Pratt measure of risk aversion," Annals of Operation Research 52, pp. 97-106, 1994.

[18] Machina, M., " 'Expected utility' analysis without the independence axiom," Econometrica 50, pp. 277-323, 1982.

[19] Machina, M., "A stronger characterization of declining risk aversion," Econometrica 50, pp. 1069-1079, 1982.

[20] Puccetti, G. and M. Scarsini, "Multivariate comonotonicity," Journal of Multivariate Analysis 101, pp. 291-304, 2010. 
[21] Quiggin, J.: "Increasing risk: another definition," In: Chikan, A. (ed.) Progress in decision, utility and risk theory. Dordrecht: Kluwer 1992.

[22] Revuz, D. and M. Yor, Continuous martingales and Brownian motion, Third Edition, Springer Verlag, 1999.

[23] Rothschild, M. and J. Stiglitz, "Increasing risk: I. A definition," Journal of Economic Theory 2, pp. 225-243, 1970.

[24] Safra, Z. and U. Segal, "Dominance axioms and multivariate nonexpected utility preferences," International Economic Review 34, pp. 321-334, 1993.

[25] Scarsini, M., "Dominance conditions for multivariate utility functions," Management Science 34, pp. 454-460, 1988.

[26] Segal, U., "Some remarks on Quiggin's anticipated utility," Journal of Economic Behavior and Organization 8, pp. 145-154, 1987.

[27] Shaked, M. and J. Shantikumar, "Two variability orders," Probability in the Engineering and Informational Sciences 12, pp. 1-23, 1998.

[28] Stiglitz, J., "Behavior towards risk with many commodities," Econometrica 37, pp. 660-667, 1969.

[29] Strassen, V., "The existence of probability measures with given marginals" Annals of Mathematical Statistics 36, pp. 423-439, 1965.

[30] Villani, C., Topics in Optimal Transportation, Providence: American Mathematical Society, 2003.

[31] Yaari, M., "Univariate and multivariate comparisons of risk aversion: a new approach," Essays in Honor of Kenneth Arrow (W. Heller, R. Starr and D. Starrett, Eds.), Cambridge University Press, pp. 173-187, 1986. 